1. Extended Data

\begin{tabular}{|c|c|c|c|}
\hline Figure \# & $\begin{array}{l}\text { Figure title } \\
\text { One sentence only }\end{array}$ & $\begin{array}{l}\text { Filename } \\
\text { This should be the } \\
\text { name the file is } \\
\text { saved as when it } \\
\text { is uploaded to our } \\
\text { system. Please } \\
\text { include the file } \\
\text { extension. i.e.: } \\
\text { Smith_ED_Fig1.j } \\
\text { pg }\end{array}$ & $\begin{array}{l}\text { Figure Legend } \\
\text { If you are citing a reference for the first time in } \\
\text { these legends, please include all new references in } \\
\text { the main text Methods References section, and } \\
\text { carry on the numbering from the main References } \\
\text { section of the paper. If your paper does not have a } \\
\text { Methods section, include all new references at the } \\
\text { end of the main Reference list. }\end{array}$ \\
\hline $\begin{array}{l}\text { Extended Data } \\
\text { Fig. } 1\end{array}$ & $\begin{array}{l}\text { Example } \\
\text { Universal } \\
\text { Spectrum } \\
\text { Identifiers with } \\
\text { explanations }\end{array}$ & $\begin{array}{l}\text { USI_Extende } \\
\text { dFigure1.eps }\end{array}$ & $\begin{array}{l}\text { Example use cases for Universal } \\
\text { Spectrum Identifiers (USIs), } \\
\text { providing a set of } 13 \text { example USIs } \\
\text { along with a brief comment on each. } \\
\text { These same } 13 \text { USIs can be easily } \\
\text { viewed as the "Example USIs" select } \\
\text { list at } \\
\text { http://proteomecentral.proteomexchan } \\
\text { ge.org/usi. Example 4c in Box } 1 \\
\text { provides the USI for the demonstrated } \\
\text { correct PSM of an ordinary } \\
\text { UniProtKB protein Q9UQ35 from } \\
\text { Mylonas et al. }{ }^{4} \text { Figure } 2 B \text { (example } \\
\text { 4d is the corresponding synthetic } \\
\text { peptide spectrum). Example } 4 \text { a in Box } \\
1 \text { provides the USI for the same } \\
\text { spectrum as example } 4 \text { c, but } \\
\text { annotated with the previously, } \\
\text { incorrectly reported HLA (Human } \\
\text { Leukocyte Antigen) peptide as } \\
\text { described in Mylonas et al. Figure } \\
\text { 2A. The non-matching synthetic } \\
\text { peptide spectrum for the incorrect } \\
\text { sequence is given as Box } 1 \text { as } \\
\text { example } 4 \text { b. The Human Proteome } \\
\text { Project }{ }^{19} \text { (HPP) has set a high bar for } \\
\text { data quality and evidence in support } \\
\text { of its goal to provide high-stringency } \\
\text { detections for all human proteins. The } \\
\text { latest version of its MS data } \\
\text { interpretation guidelines } 3.0^{20} \text { have } \\
\text { set a requirement that key detection } \\
\text { claims of proteins not previously seen } \\
\text { via MS must be accompanied by USIs } \\
\text { referencing the key spectra for each }\end{array}$ \\
\hline
\end{tabular}




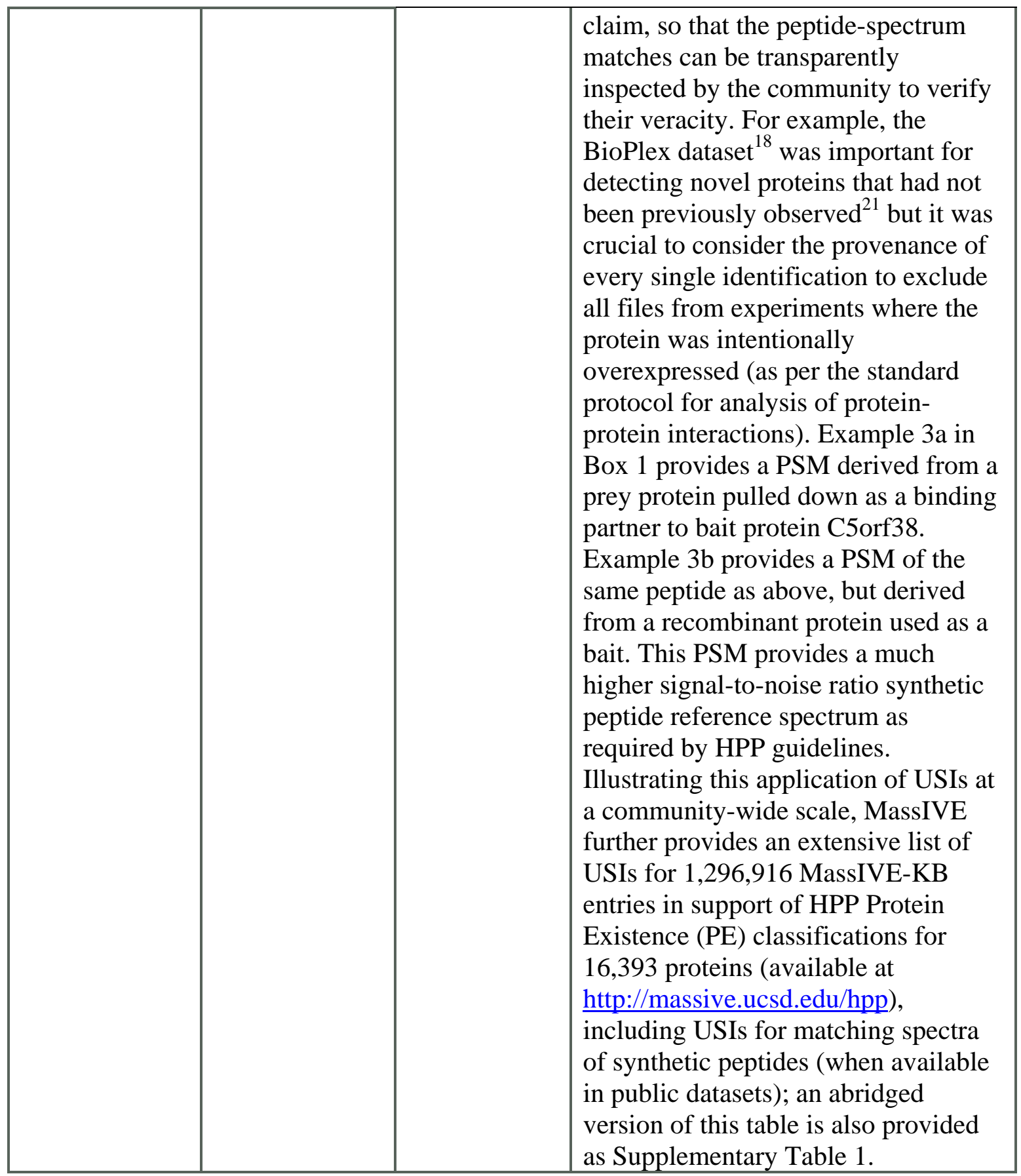

3 2. Supplementary Information:

\begin{tabular}{|l|l|l|l|}
\hline Item & Present? & $\begin{array}{l}\text { Filename } \\
\text { This should be the } \\
\text { name the file is } \\
\text { saved as when it is } \\
\text { uploaded to our } \\
\text { system, and should } \\
\text { include the file } \\
\text { extension. The }\end{array}$ & $\begin{array}{l}\text { A brief, numerical description of file } \\
\text { contents. } \\
\text { i.e.: Supplementary Figures 1-4, Supplementary } \\
\text { Discussion, and Supplementary Tables 1-4. }\end{array}$ \\
\hline
\end{tabular}




\begin{tabular}{|c|c|c|c|}
\hline & & $\begin{array}{l}\text { extension must be } \\
\text {.pdf }\end{array}$ & \\
\hline $\begin{array}{l}\text { Supplementary } \\
\text { Information }\end{array}$ & yes & $\begin{array}{l}\text { USI_1.6_Suppl } \\
\text { ementary_Infor } \\
\text { mation.pdf }\end{array}$ & Supplementary Figures S1 - S6 \\
\hline Reporting Summary & yes & $\begin{array}{l}\text { nr-reporting- } \\
\text { summary.pdf }\end{array}$ & \\
\hline
\end{tabular}

B. Additional Supplementary Files

\begin{tabular}{|l|l|l|}
\hline & $\begin{array}{l}\text { Number } \\
\text { If there are multiple files of the same } \\
\text { type this should be the numerical } \\
\text { indicator. i.e. "1" for Video 1, "2" for } \\
\text { Video 2, etc. }\end{array}$ & $\begin{array}{l}\text { Filename } \\
\text { This should be the name the file is } \\
\text { saved as when it is uploaded to our } \\
\text { system, and should include the file } \\
\text { extension. i.e.: Smith___mov } \\
\text { Supplementary_Video_1.mov }\end{array}$ \\
\hline Supplementary Table & 1 & $\begin{array}{l}\text { USI_1.6_Supplementary_ta } \\
\text { ble1.xlsx }\end{array}$ \\
\hline
\end{tabular}

\section{Universal Spectrum Identifier for mass spectra}

9 Eric W. Deutsch ${ }^{1, *}$, Yasset Perez-Riverol ${ }^{2}$, Jeremy Carver ${ }^{3}$, Shin Kawano ${ }^{4}$, Luis

10 Mendoza $^{1}$, Tim Van Den Bossche ${ }^{5,6}$, Ralf Gabriels ${ }^{5,6}$, Pierre-Alain Binz ${ }^{7}$, Benjamin

11 Pullman $^{3}$, Zhi Sun ${ }^{1}$, Jim Shofstahl ${ }^{8}$, Wout Bittremieux ${ }^{9,10}$, Tytus D. Mak ${ }^{11}$, Joshua

12 Klein ${ }^{12}$, Yunping Zhu ${ }^{13}$, Henry Lam ${ }^{14}$, Juan Antonio Vizcaíno ${ }^{2}$, and Nuno Bandeira ${ }^{3,9, *}$

$14{ }^{1}$ Institute for Systems Biology, 401 Terry Ave N, Seattle, WA, 98109, USA

$15{ }^{2}$ European Molecular Biology Laboratory, European Bioinformatics Institute (EMBL-

16 EBI), Wellcome Trust Genome Campus, Hinxton, Cambridge CB10 1SD, United

17 Kingdom.

$18{ }^{3}$ Center for Computational Mass Spectrometry, Department of Computer Science and

19 Engineering, Skaggs School of Pharmacy and Pharmaceutical Sciences, University of

20 California, San Diego, 92093-0404, USA

$21{ }^{4}$ Toyama University of International Studies, 930-1292 Toyama, Japan

$22 \quad{ }^{5}$ VIB - UGent Center for Medical Biotechnology, VIB, Ghent, Belgium

$23{ }^{6}$ Department of Biomolecular Medicine, Faculty of Medicine and Health Sciences,

24 Ghent University, Ghent, Belgium

$25{ }^{7}$ Clinical Chemistry Service, Lausanne University Hospital, 1011 Lausanne, Switzerland

$26{ }^{8}$ Thermo Fisher Scientific, 355 River Oaks Parkway San Jose, CA 95134, USA

$27{ }^{9}$ Skaggs School of Pharmacy and Pharmaceutical Sciences, University of California San

28 Diego, La Jolla, CA 92093, USA 
${ }^{10}$ Department of Computer Science, University of Antwerp, 2020 Antwerp, Belgium

$30 \quad{ }^{11}$ Mass Spectrometry Data Center, National Institute of Standards and Technology, 100

31 Bureau Drive, Gaithersburg, MD 20899, USA

$32{ }^{12}$ Program for Bioinformatics, Boston University, Boston, MA 02215, USA

$33{ }^{13}$ National Center for Protein Sciences (Beijing), Beijing Institute of Lifeomics, \#38, Life

34 Science Park, Changping District, Beijing 102206, China

$35{ }^{14}$ Department of Chemical and Biological Engineering, the Hong Kong University of

36 Science and Technology, Clear Water Bay, Hong Kong

*Address correspondence to:

39 Email: edeutsch@systemsbiology.org, Phone: 206-732-1200, Fax: 206-732-1299.

40 Email: bandeira@ucsd.edu, Phone: 858-534-8666

43 Keywords: Proteomics Standards Initiative, PSI, mass spectrometry, proteomics,

44 Universal Spectrum Identifier, USI, standards.

45 Mass spectra provide the ultimate evidence for supporting the findings of mass

46 spectrometry (MS) proteomics studies in publications, and it is therefore crucial to be

47 able to trace the conclusions back to the spectra. The Universal Spectrum Identifier (USI)

48 provides a standardized mechanism for encoding a virtual path to any mass spectrum

49 contained in datasets deposited to public proteomics repositories. USIs enable greater

50 transparency for providing spectral evidence, with more than 1 billion USI identifications

51 from over 3 billion spectra already available through ProteomeXchange (PX)

52 repositories.

54 MS is a major analytical tool for the fields of proteomics and metabolomics among

55 others, enabling high throughput identification and quantitation of millions of different

56 analytes including e.g. peptides and small molecules. The availability of proteomics data

57 in the public domain has increased dramatically in recent years due to the wide adoption

58 of open data practices in the field, which has been enabled by the reliability of the public 
59 resources that are part of the ProteomeXchange ${ }^{1}$ consortium. But while the public

60 availability of the raw mass spectrometry data creates the possibility for scientists to

61 directly inspect whether the data support published results as claimed in the

62 corresponding papers, this remains difficult to implement in practice because there is no

63 standard mechanism supported by the repositories to directly reference individual mass

64 spectra whose interpretations are crucial to the published results. As it stands today, the

65 key spectral evidence for biological studies lacks 'FAIRness' (i.e. Findable, Accessible,

66 Interoperable and Reusable) $)^{2}$ and is at best found as a figure in the manuscript or in the

67 supplementary information, which is typically of low quality and does not provide the

68 connection to the raw data (as is required for inspection of the results for the correctness

69 of the corresponding claims). This need becomes even more pressing as the promise of

70 open data for the advancement of science begins to be realized by data reanalyses

71 revealing multiple cases where the data do not support published results ${ }^{3,4}$. We thus

72 propose a new standardized mechanism supported by PX proteomics repositories for

73 referring to specific spectra of high importance, suitable for citation of data in published

74 manuscripts, as well as for supporting both human interactions and automated access to

75 the data via application programming interfaces (APIs).

77 A related mechanism for identifying identical metabolomics mass spectra across

78 repositories has been proposed as the SPLASH identifier ${ }^{5}$, an algorithm-generated hash

79 of the spectrum data. SPLASH was designed to determine if the same exact reference

80 spectrum is present in multiple metabolomics resources, since an identical spectrum

81 generates an identical SPLASH identifier. However, (i) SPLASH was based on statistics 
82 of metabolomics spectra that do not apply to proteomics, (ii) requires pre-computing

83 billions of hashes in advance to make them findable and (iii) uses hashes that are not

84 interpretable by humans, and thus is not suitable as a general-purpose identifier for all

85 spectra in proteomics data sets deposited to public repositories.

87 Here we present an alternative approach using a concatenated multi-part key that

88 specifies the collection, MS run, and index information needed to locate a particular mass

89 spectrum in a repository, defining keys that researchers can easily compose without

90 requiring any special hashing algorithms. This concept has been developed by the Human

91 Proteome Organization ${ }^{6}$ (HUPO) Proteomics Standards Initiative ${ }^{7}$ (PSI) as the USI. It

92 provides a standardized mechanism for identifying a mass spectrum via an abstract path

93 to its location that can be easily mapped to a local path at each resource that contains the

94 spectrum.

95

96 The full specification document describing in detail all aspects of version 1.0 of this

97 standard as well as future updates to this specification and information about current

98 implementations with examples are available on the USI page at the PSI web site at

99 http://psidev.info/usi. In this letter, we provide a brief overview of the USI's components

100 and describe prominent current applications.

103 The USI is a multipart key separated by colon characters. The basic form of the USI has

104 five components with an optional sixth as depicted in Figure 1. The first component is the 105 prefix "mzspec" to make clear that the following string is a USI; mzspec is a registered 
namespace at identifiers.org ${ }^{8}$ to aid in automated mapping of a USI string to a URL

107 (https://registry.identifiers.org/registry/mzspec). The second component is the collection

108 identifier, initially intended to be a PX dataset (PXD) identifier ${ }^{9}$, but can be extended to

109 other types of collection identifiers, and can take on the special value of USI000000 as a

110 placeholder for subsequent dataset submission. The third component is the MS run name,

111 typically the root name of the raw vendor format file or mzML ${ }^{10}$ file, either with or

112 without the file extension, depending on the use case as fully described in the

113 specification. The fourth component is a type flag to indicate how to interpret the fifth

114 component; currently allowed values are "scan”, “index”, or "nativeId” (as defined in the

115 mzML 1.1.0 specification available at http://psidev.info/mzML). The fifth component is

116 the index value (based on the flag type) within the specified MS run. While these five

117 components can define the abstract path to a spectrum, one additional (sixth) component

118 can specify an interpretation of the spectrum when one is known or proposed. There are

119 many nuances to the specification and the usage of these components that are described

120 in detail in the full specification document.

123 The spectrum interpretation, depicted as a simple peptide sequence and charge in Figure

124 1, can become quite complex when taking into account different types of mass

125 modifications, adducts, and non-peptidic molecules, among other scenarios. An entire

126 separate PSI specification, called ProForma 2.0, has been developed to define the rules

127 for representing complex peptidoforms and proteoforms in a standardized manner. The

128 current version is available at the PSI web page http://psidev.info/proforma. 
130 There are already several implementations of the USI, and more are emerging. At the 131 spectrum viewer applications of the PX resources PRIDE ${ }^{11}$, PeptideAtlas ${ }^{12}$, MassIVE ${ }^{13}$,

$132 \mathrm{jPOST}^{14}$, and $\mathrm{iProX}{ }^{15}$, the USI is displayed for each spectrum viewed when the data set

133 containing the spectrum is associated with a PXD identifier, and these resources also

134 provide a text box in which a USI can be pasted in order to display the requested

135 spectrum (see Online Methods for listing of URLs and a description of the

136 implementations at each site). For convenience, the ProteomeCentral resource of PX

137 implements a single endpoint at http://proteomecentral.proteomexchange.org/usi/ that

138 reaches out to all participating partners to fetch spectra for a provided USI if available at

139 any resource. It also provides USI validator functionality, which checks that the format of

140 the USI complies with the USI specification. An overview of the USI ecosystem is

141 depicted in Figure 2.

142

143 Since the USI is designed as a virtual path to a spectrum based on a set of rules and

144 conventions, there is no need to compute hashes for billions of existent spectra or newly

145 generated spectra like in the case of the SPLASH identifier. Implementing repositories

146 need only map the virtual path components (collection, MS run, indexType) to their local

147 storage system to make the spectra findable and accessible. As a key point, USIs can be

148 retroactively determined for spectra deposited long ago to a PX repository (the spectrum

149 depicted in Figure 1 was deposited in 2013). Furthermore, authors of new publications

150 can easily construct and provide within their articles references to key spectra that

151 support their conclusions by knowing the collection identifier (typically known as a

152 manuscript is being submitted), the MS run filename, and spectrum scan number, which 
153 are usually determinable from within most analysis environments. PX repositories also

154 provide various tools to assist with the process of generating USIs for dataset spectra (see

155 Online Methods). Within some proteomics analysis workflows, the provenance

156 information about the original scan numbers is unfortunately lost during processing, but

157 the importance of provenance is now well understood and any such loss of provenance

158 should be remedied in future software revisions. See Extended Data Figure 1 for a set of

15912 example USIs with extended descriptions.

160

161 USI provides a standardized mechanism for encoding a virtual path to any spectrum

162 contained in data sets deposited to public repositories or contained in public spectral

163 libraries. Current PX data repositories have implemented support for USIs as part of the

164 submission or browsing process as described in the Online Methods. It has been primarily

165 designed for the proteomics community, but could easily be adopted by the metabolomics

166 community (and potentially others) if there is a will to do so (the current USI

167 specification already supports non-proteomics spectra but additional guidelines would be

168 needed for instance to represent non-proteomics identifications). It enables greater

169 transparency and data 'FAIRness' ${ }^{2}$ for providing spectral evidence in support of key

170 findings in publications and public data, and provides a standardized mechanism for

171 communicating identifiers of specific spectra among software applications. We thus

172 expect that USIs will become a fundamental mechanism supporting open science across

173 all fields of research supported by MS data.

174

175 


\section{$176 \quad$ Notes}

178 The authors declare no competing financial interest.

179

180 Acknowledgements

182 This work was funded in part by the National Institutes of Health grants R01GM087221, 183 R24GM127667, 1R01LM013115 and P41GM103484, and National Science Foundation 184 grants 1933311, 1922871 and ABI 1759980. JAV wants to acknowledge Wellcome Trust 185 grant number 208391/Z/17/Z, BBSRC BB/S01781X/1, BB/P024599/1 and the partnering 186 grants BB/N022440/1 and BB/N022432/1. SK wants to acknowledge the Database

187 Integration Coordination Program from the National Bioscience Database Center, Japan

188 Science and Technology Agency [18063028] and the Japan Society for the Promotion of

189 Science KAKENHI [JP20H03245]. We also would like to acknowledge the Research

190 Foundation - Flanders (SB grant 1S90918N to TVDB; SB grant 1S50918N to RG; WB is 191 a postdoctoral researcher).

192

\section{Data availability statement}

195 No datasets were generated or analyzed during the current study. 


\section{Code availability statement}

198

199 No custom code was used to analyze data, but code used to fetch spectra using USIs is

200 publicly available in GitHub at https://github.com/proteomexchange/proteomecentral.

201

202

203 References

204

205 1. Deutsch, E. W. et al. The ProteomeXchange consortium in 2020: enabling 'big data'

206 approaches in proteomics. Nucleic Acids Res. 48, D1145-D1152 (2020).

207 2. Wilkinson, M. D. et al. The FAIR Guiding Principles for scientific data management

208 and stewardship. Sci. Data 3, 160018 (2016).

209 3. Ezkurdia, I., Vázquez, J., Valencia, A. \& Tress, M. Analyzing the first drafts of the

210 human proteome. J. Proteome Res. 13, 3854-3855 (2014).

211 4. Mylonas, R. et al. Estimating the Contribution of Proteasomal Spliced Peptides to the

212 HLA-I Ligandome. Mol. Cell. Proteomics MCP 17, 2347-2357 (2018).

213 5. Wohlgemuth, G. et al. SPLASH, a hashed identifier for mass spectra. Nat.

214 Biotechnol. 34, 1099-1101 (2016).

215 6. Hanash, S. \& Celis, J. E. The Human Proteome Organization: a mission to advance

216 proteome knowledge. Mol. Cell. Proteomics MCP 1, 413-414 (2002).

217 7. Deutsch, E. W. et al. Development of data representation standards by the human

218 proteome organization proteomics standards initiative. J. Am. Med. Inform. Assoc.

219 JAMIA 22, 495-506 (2015). 
220 8. Wimalaratne, S. M. et al. Uniform resolution of compact identifiers for biomedical 221 data. Sci. Data 5, 180029 (2018).

222 9. Deutsch, E. W. et al. The ProteomeXchange consortium in 2017: supporting the 223 cultural change in proteomics public data deposition. Nucleic Acids Res. 45, D1100224 D1106 (2017).

225 10. Martens, L. et al. mzML--a community standard for mass spectrometry data. Mol. 226 Cell. Proteomics MCP 10, R110.000133 (2011).

227 11. Perez-Riverol, Y. et al. The PRIDE database and related tools and resources in 2019:

228 improving support for quantification data. Nucleic Acids Res. 47, D442-D450 (2019).

229 12. Desiere, F. et al. The PeptideAtlas project. Nucleic Acids Res. 34, D655-658 (2006).

230 13. Wang, M. et al. Assembling the Community-Scale Discoverable Human Proteome. $231 \quad$ Cell Syst. 7, 412-421.e5 (2018).

232 14. Moriya, Y. et al. The jPOST environment: an integrated proteomics data repository 233 and database. Nucleic Acids Res. 47, D1218-D1224 (2019).

234 15. Ma, J. et al. iProX: an integrated proteome resource. Nucleic Acids Res. 47, D1211235 D1217 (2019).

236 16. Kim, M.-S. et al. A draft map of the human proteome. Nature 509, 575-581 (2014).

237 17. Zhou, J.-Y. et al. Quality Assessments of Long-Term Quantitative Proteomic

238 Analysis of Breast Cancer Xenograft Tissues. J. Proteome Res. 16, 4523-4530 $239 \quad$ (2017).

240 18. Huttlin, E. L. et al. The BioPlex Network: A Systematic Exploration of the Human 241 Interactome. Cell 162, 425-440 (2015). 
242 19. Omenn, G. S. et al. Research on The Human Proteome Reaches a Major Milestone:

$243>90 \%$ of Predicted Human Proteins Now Credibly Detected, according to the HUPO

$244 \quad$ Human Proteome Project. J. Proteome Res. (2020)

245 doi:10.1021/acs.jproteome.0c00485.

246 20. Deutsch, E. W. et al. Human Proteome Project Mass Spectrometry Data

247 Interpretation Guidelines 3.0. J. Proteome Res. 18, 4108-4116 (2019).

248 21. Pullman, B. S., Wertz, J., Carver, J. \& Bandeira, N. ProteinExplorer: A Repository-

249 Scale Resource for Exploration of Protein Detection in Public Mass Spectrometry

250 Data Sets. J. Proteome Res. 17, 4227-4234 (2018).

251

252 
Figure 1: (A) Graphical overview of the general format of the USI, including the mzspec prefix, the collection component, the MS run component, the indexType, the scan number, once with and once without the optional spectrum interpretation. (C) Visual representation of this spectrum (mzspec:PXD000561:Adult_Frontalcortex_bRP_Elite_85_f09:scan:17555:VLHPLEGA VVIIFK/2) in ProteomeCentral, accompanied with the ion table indicating the $\mathrm{m} / \mathrm{z}$ values

\section{Online Methods}

List of resources and their URLs that allow a user to copy-paste a USI into the web page and visualize the spectrum if it is available

\begin{tabular}{|l|l|}
\hline Resource & URL \\
\hline ProteomeCentral & $\underline{\text { http://proteomecentral.proteomexchange.org/usi/ }}$ \\
\hline jPOST & $\underline{\text { https://repository.jpostdb.org/spectrum/ }}$ \\
\hline MassIVE & $\underline{\text { http://massive.ucsd.edu/ProteoSAFe/usi.jsp }}$ \\
\hline PeptideAtlas & $\underline{\text { https://db.systemsbiology.net/sbeams/cgi/PeptideAtlas/ShowObservedSpectrum }}$ \\
\hline PRIDE & $\underline{\text { https://www.ebi.ac.uk/pride/archive/spectra }}$ \\
\hline ProteomicsDB & $\underline{\text { https://www.proteomicsdb.org/use/ }}$ \\
\hline GNPS (metabolomics) & $\underline{\text { https://gnps.ucsd.edu/ProteoSAFe/gnps usi.jsp }}$ \\
\hline iProX & $\underline{\text { https://www.iprox.org/page/spectrum.html }}$ \\
\hline
\end{tabular}




\section{USI Implementation notes from each resource}

276 ProteomeCentral

277 ProteomeCentral hosts a web-based USI validator and resolver at 278 http://proteomecentral.proteomexchange.org/usi/. Users of this web page may paste a USI 279 into a text box and choose either to validate or fetch the spectrum/PSM encoded in the 280 USI. Validation is performed by a back-end service that parses the supplied USI and 281 decomposes it into its various components and ensures that they are well formed and 282 follow the rules of the specification. Collection identifiers are checked to ensure that they encode a supported namespace, but not that they are within a valid range. MS runs components are separated but not checked as essentially any string is permitted. The indexType is checked for a valid value. The indexNumber is separated but not checked against the relevant file to ensure that it is within range. The interpretation string is parsed to ensure it is well formed and that controlled vocabulary (CV) terms are present in the relevant CVs. The service returns the decomposed and interpreted USI and a flag to indicate if it is valid or not. A well-formed USI may not actually correspond to a spectrum, but this cannot be determined without asking all resources to look up the USI.

The USI lookup function first validates the provided USI as described above, and then, if valid, sends the USI to all participating resource services that can provide spectra given a USI. USI lookups are performed in parallel within the JavaScript. A result table depicts the results from the participating resources as they arrive in real time. The first spectrum to return from one of the resources is sent to an embedded Lorikeet spectrum viewer in the page with basic defaults for an HCD MS/MS spectrum, along with the peptide interpretation, if provided. The user may click on various hyperlinks in the table to see the spectrum obtained from different resources, link out to the resources themselves, where the user can explore the spectrum using the native tools at the resource, as well as access additional information about the returned spectrum data. The USI lookup service may be triggered automatically without required copy-paste with a URL http://proteomecentral.proteomexchange.org/usi/?usi=<USI $>$ where the $<$ USI $>$ is replaced with an actual USI, e.g. http://proteomecentral.proteomexchange.org/usi/?usi=mzspec:PXD000561:Adult_Frontal cortex_bRP_Elite_85_f09:scan:17555:VLHPLEGAVVIIFK/2.

\section{jPOST}

jPOST provides a USI resolver at https://repository.jpostdb.org/spectrum/. Currently there are 27M spectra corresponding to USIs. Spectra submitted by "Complete Submission" are basically available via USI. In addition, spectra submitted to PX partners 311 other than jPOST will also provide USI from jPOST in the case of frequently used 312 datasets. 


\section{MassIVE}

315 The main MassIVE interface for resolution of USIs is available at 316 https://massive.ucsd.edu/ProteoSAFe/usi.jsp. At the time of writing, MassIVE can 317 resolve USIs for over $>3.3$ billion spectra in $>10,000$ datasets. In addition, MassIVE can 318 also resolve USIs to >580 million Peptide Spectrum Matches (PSMs), including both 319 PSMs that were included in complete dataset submissions (reported with PXD and MSV 320 accessions) and PSMs derived by MassIVE (and third-party) reanalysis of public datasets 321 (reported with RPXD and RMSV accessions). As illustrated in Figure S1, USIs can be 322 entered in a text box and can be used to search MassIVE datasets in four different ways. 323 Each of the four different types of search is accompanied by a small blue icon containing 324 a short description of each type of search, as well as listing an example USI illustrating 325 the types of results for each search.

The first option ("Search MassIVE spectra”, also illustrated in Figure S1) is to search for the dataset and spectrum file(s) as specified in the USI mzspec:PXD000561:Adult_Frontalcortex_bRP_Elite_85_f09:scan:17555:VLHPLEGAV $\underline{V I I F K / 2}$. If successful, this search returns a table with one row per spectrum file with a matching file name. Clicking on the spectrum icon (on the left) for rows corresponding to spectrum files in standard open formats will open a new panel allowing for visualization and interactive exploration of the USI spectrum. If a peptide annotation is included in the USI then it is used to label and color spectrum peaks according to the theoretical masses of the standard fragment ion types.

The second option ("Search all MassIVE PSMs") is to search all of MassIVE for other possible identifications for the spectrum indicated in the USI. Since spectrum files can be resubmitted in multiple datasets and reanalyzed in multiple RPXD/RMSV reanalyses, this option searches for all occurrences of MassIVE PSMs matching the USI spectrum file and spectrum identifier (e.g., scan number). The example in Figure S2(a) shows that the initial spectrum identification (mzspec:PXD002041:TCGA-AA-3518-01A11_W_VU_20120915_A0218_3F_R_FR04:scan:9649:ADNQRLKDENGALIR/2)

reported in a proteogenomics search for a CPTAC dataset is actually a worse match to the spectrum than the alternative identification to a different peptide found in MassIVE reanalyses with a common $\mathrm{N}$-term modification of +28 Da (likely Formylation, a common sample handling artefact), a type of modification that was not considered in the original paper.

The third option ("Search MassIVE-KB reference spectra") searches the MassIVE Knowledge Base (MassIVE-KB) for reference spectra for the same peptide as in the USI. Reference spectra can include spectra of synthetic peptides as well as spectra selected as representatives in the MassIVE-KB spectral library. As shown in Figure S2(b), the spectrum in the USI mzspec:PXD004452:20150410_QE3_UPLC9_DBJ_SA_46fractions_Rep1_18:scan:2873

356 :PAPTLEEEKIR/2 was incorrectly identified to a peptide whose reference spectrum 
shows a distinct fragmentation pattern than that of the synthetic peptide reported in this search.

The fourth option (“Search Protein Explorer proteins”) searches MassIVE's Protein Explorer for human proteins containing the USI peptide sequence. As shown in Figure

$$
\begin{aligned}
& \text { S2(c), the } \\
& \text { mzspec:PXD000865:00644_H11_P004899_B0P_A00_R1:scan:3727:PAGDGTFQK/2 }
\end{aligned}
$$

\section{PeptideAtlas}

374 PeptideAtlas provides access to over 1 billion spectra via the USI mechanism. Users can https://db.systemsbiology.net/sbeams/cgi/PeptideAtlas/ShowObservedSpectrum and copy-paste a USI. If the dataset is housed at PeptideAtlas, then the system dynamically finds the location of the dataset on PeptideAtlas disks, finds the mzML.gz file corresponding to the requested MS run, and then extracts the requested spectrum from the mzML.gz file. Part of the PeptideAtlas processing pipeline is to download raw vendor files from the original hosting repositories and convert them to mzML.gz via msconvert or ThermoRawFileReader and these are all stored in an accessible manner. The extracted spectrum is then displayed with the requested annotation embedded in the USI if there is one.

Additionally, as users are browsing the PeptideAtlas web interface, if they click on a link to view a PSM in a PeptideAtlas build, if the dataset has a PXD identifier (not all datasets in PeptideAtlas do), then a USI for that PSM is created and displayed above the spectrum.

392 At the time of writing, the PRIDE database provides access to over 540 million peptidespectrum matches (PSMs) originally submitted by researchers in the PRIDE Archive Spectra resource (https://www.ebi.ac.uk/pride/archive/spectra). Users can search by USIs, which enables them to find specific PSMs from submitted data. 
including peptide sequences, post-translational modifications, search engine scores, charge, and two additional columns that highlight whether the PSM has passed or not the original analysis threshold and PRIDE internal pipelines thresholds (e.g. PSM FDR < $0.1)$.

The accession column in the result table provides a direct link to the project centric page which contains the information of the original submitted dataset, including the results details page. If users perform a search using a USI (e.g. https://www.ebi.ac.uk/pride/archive/spectra?usi=mzspec:PXD000966:CPTAC_CompRef _00_iTRAQ_12_5Feb12_Cougar_11-1011.mzML:scan:11850:[UNIMOD:214]YYWGGLYSWDMSK[UNIMOD:214]/2), the corresponding spectra will be shown in the spectrum panel below in the same page.

It is possible to access and visualise any PSM included in a publicly available "complete" PRIDE dataset. To do that, users need to go to https://www.ebi.ac.uk/pride/archive/spectra and search for a particular peptide sequence.

For instance, for the peptide sequence WQLVGITSWGEGCAR, the resulting URL is: https://www.ebi.ac.uk/pride/archive/spectra?peptideSequence=WQLVGITSWGEGCAR (Figure S5). At that point it is possible to select all PSMs containing that particular sequence in a given dataset (Figure S6).

\section{ProteomicsDB (Universal Spectrum Explorer)}

The ProteomicsDB team has developed a web tool called Universal Spectrum Explorer (USE - https://www.proteomicsdb.org/use/)[DOI:10.1101/2020.09.08.287557v1]; to query and visualize public spectra using USIs from multiple archives including PRIDE and PeptideAtlas. In addition, USE can retrieve real-time prediction of tandem mass spectra from the deep learning framework Prosit. The query spectra from public USI or provided by the user can be exported from USE as editable scalable high quality vector graphics

(e.g., https://www.proteomicsdb.org/use/?usi=mzspec:PXD015890:18May18_Olson_WT2.raw \%20(F001551).mzid_18May18_Olson_WT2.raw_(F001551).MGF:index:6913:AEAEA QAEELSFPR/2\&usi_origin=pride). 


\section{GNPS (metabolomics)}

445 GNPS hosts the Metabolomics Spectrum Resolver tool at

446 https://gnps.ucsd.edu/ProteoSAFe/gnps_usi.jsp [DOI:10.1101/2020.05.09.086066] to

447 access metabolomics mass spectra available in MassIVE/GNPS and alternative

448 metabolomics data repositories. Besides spectra deposited to GNPS

449 [DOI:10.1038/nbt.3597], the Metabolomics Spectrum Resolver builds upon the USI

450 standard to resolve spectra from other popular metabolomics data resources, such as

451 MetaboLights [DOI:10.1093/nar/gks1004], Metabolomics Workbench

452 [DOI:10.1093/nar/gkv1042], MassBank [DOI:10.1002/jms.1777], MoNA, and MS2LDA

453 [DOI:10.1093/bioinformatics/btx582] to provide a universal interface to more than 450

454 million metabolomics spectra. The Metabolomics Spectrum Resolver can display

455 individual spectra or display two spectra in a mirror plot to evaluate their similarity.

456 Additionally, it supports linking back to the source spectra in their original data resources

457 to allow users to explore the original context of the spectra and computes SPLASH

458 identifiers [DOI:10.1038/nbt.3689] to enable comparisons of spectra across different

459 resources.

460

461

462

463

\section{4 iProX}

465 iProX provides USI search and display at http://www.iprox.org/page/spectrum.html for 6

466 million spectra in the HBase. The result file of the "complete submission" project in

467 iProX are parsed into spectra records uniquely marked by USIs and stored in the HBase.

468 There is also a backup mechanism to ensure data reliability. 
473 Figure 2. Graphical depiction of USI application ecosystem. Members of the community 474 can uniquely identify spectra from journal articles and other sources using USIs. A USI

475 can be resolved potentially at any of several different repositories that store datasets, or 476 spectra can be obtained and viewed using independent applications, such as at 477 ProteomeCentral, which store no spectra themselves, but can fetch spectral data from 478 repositories using USIs. Hundreds of millions of peptide-spectrum matches (PSMs) and 479 spectra without matches are accessible via USIs at the various repositories. PSMs or 480 spectra can even be uniquely identified with QR (Quick Response) codes. 
Extended Data Figure 1. Example use cases for Universal Spectrum Identifiers (USIs), providing a set of 13 example USIs along with a brief comment on each. These same 13 USIs can be easily viewed as the "Box 1 example USIs" select list at

488 http://proteomecentral.proteomexchange.org/usi. Example 4c in Box 1 provides the USI for the demonstrated correct PSM of an ordinary UniProtKB protein Q9UQ35 from

Example 4a in Box 1 provides the USI for the same spectrum as example 4c, but annotated with the previously, incorrectly reported HLA (Human Leukocyte Antigen) peptide as described in Mylonas et al. Figure 2A. The non-matching synthetic peptide spectrum for the incorrect sequence is given as Box 1 as example $4 \mathrm{~b}$. The Human Proteome Project ${ }^{19}$ (HPP) has set a high bar for data quality and evidence in support of its goal to provide high-stringency detections for all human proteins. The latest version of its MS data interpretation guidelines $3.0^{20}$ have set a requirement that key detection claims of proteins not previously seen via MS must be accompanied by USIs referencing the key spectra for each claim, so that the peptide-spectrum matches can be transparently inspected by the community to verify their veracity. For example, the BioPlex dataset ${ }^{18}$ was important for detecting novel proteins that had not been previously observed ${ }^{21}$ but it was crucial to consider the provenance of every single identification to exclude all files from experiments where the protein was intentionally overexpressed (as per the standard protocol for analysis of protein-protein interactions). Example 3a in Box 1 provides a PSM derived from a prey protein pulled down as a binding partner to bait protein C5orf38. Example 3b provides a PSM of the same peptide as above, but derived from a recombinant protein used as a bait. This PSM provides a much higher signal-to-noise ratio synthetic peptide reference spectrum as required by HPP guidelines. Illustrating this application of USIs at a community-wide scale, MassIVE further provides an extensive list of USIs for 1,296,916 MassIVE-KB entries in support of HPP Protein Existence (PE) classifications for 16,393 proteins (available at http://massive.ucsd.edu/hpp), including USIs for matching spectra of synthetic peptides (when available in public datasets); an abridged version of this table is also provided as Supplementary Table 1. 
(A) General format

mzspec: $<$ collection $>:<$ msRun $>:<$ indexType $>:<$ indexNumber $>:<$ optionalInterpretation $>$

(B) Dataset spectrum example using native scan number, with and without optional interpretation mzspec:PXD000561:Adult_Frontalcortex_bRP_Elite_85_f09:scan:17555

mzspec:PXD000561:Adult_Frontalcortex_bRP_Elite_85_f09:scan:17555:VLHPLEGAVVIIFK/2

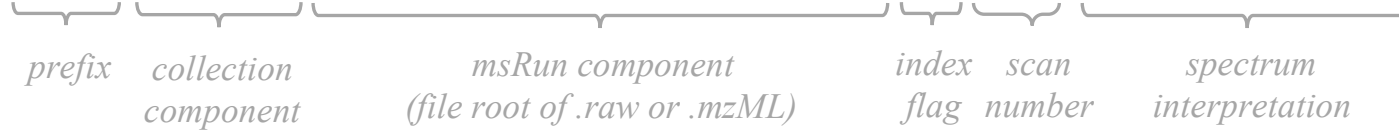

(C) Visualization in ProteomeCentral

VLHPLEGAVVIIFK, MH+ 1534.9356, $\mathrm{m} / \mathrm{z} 767.9714$

File: VLHPLEGAVVIIFK/2, Scan: 17555, Exp. m/z: 767.9700, Charge: 2

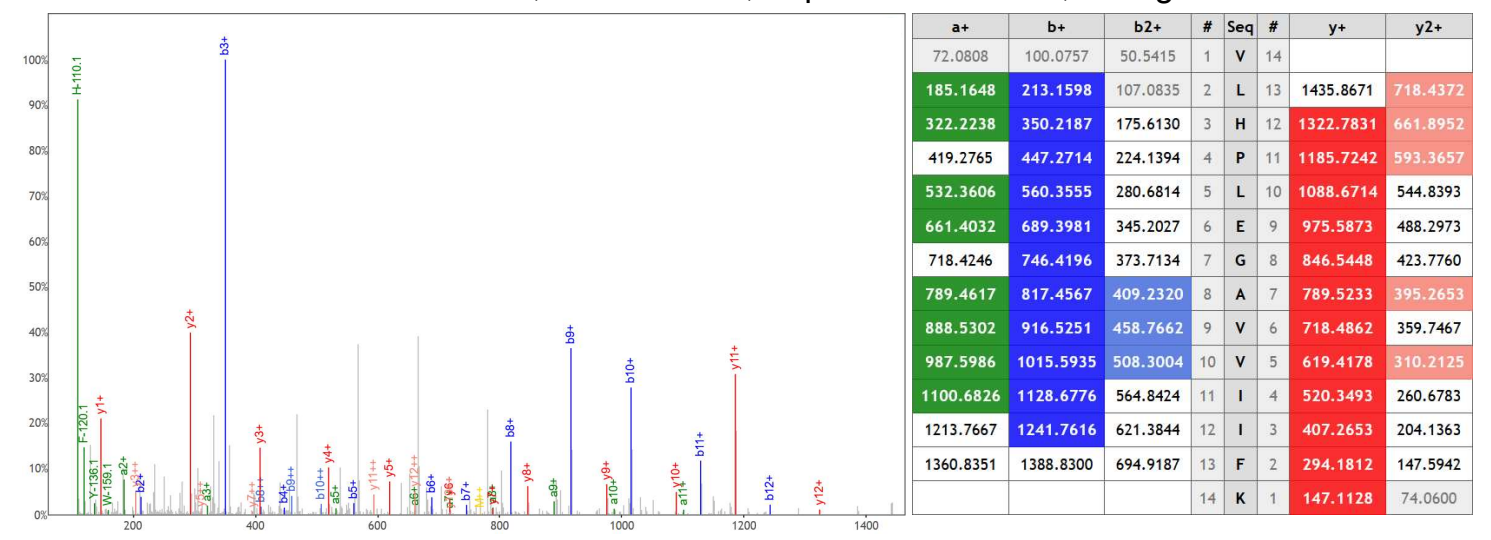




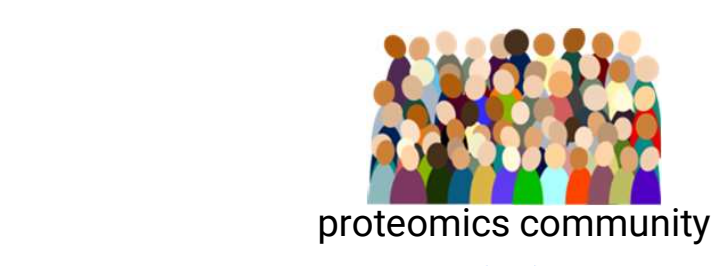

\section{UNIVERSAL SPECTRUM IDENTIFIER}

Journal Article

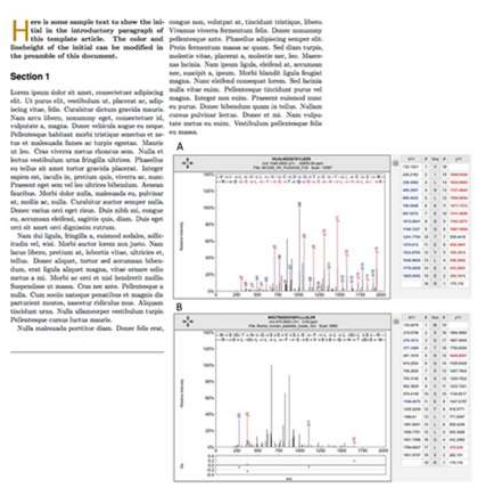

mzspec:PXD000561:Adult_Frontalcortex_bRP_Elite_85_f09:scan:17555

VLHPLEGAVVIIFK, MH+ 1534.9356, m/z 767.9714 File: VLHPLEGAVVIIFK/2, Scan: 17555, Exp. m/z: 767.9700, Charge: 2

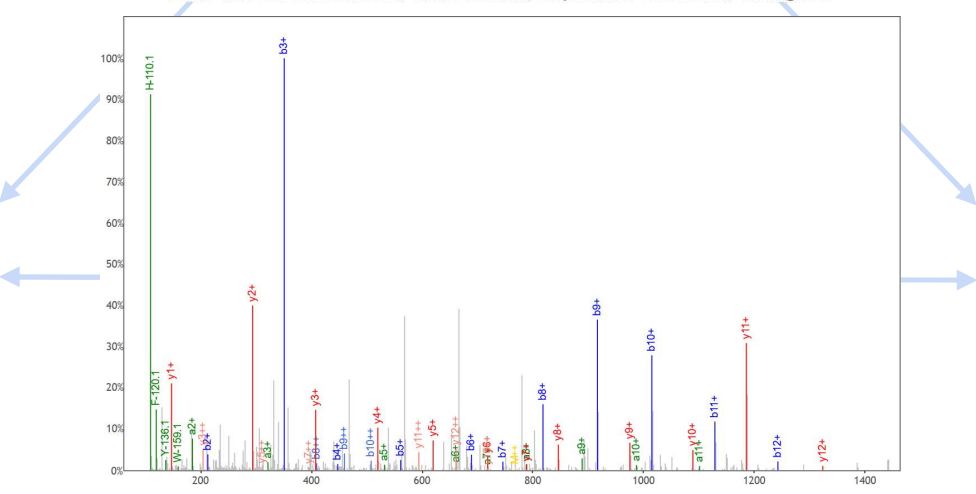

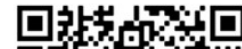

कis

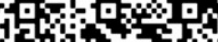

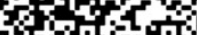

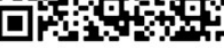

Preteome rchange

PRIDE Archive

470 M PSMs $470 \mathrm{M}$ spectra

\section{Mass/VE $\quad 580 \mathrm{MPSM}$}

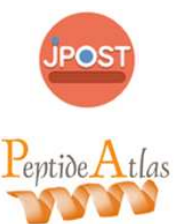

27 M PSMs $27 \mathrm{M}$ spectra

334 M PSMs 1 B spectra 
Case 1: Typical identification of an unmodified peptide in support of protein identification (one spectrum, simple interpretation, no mass modifications)

mzspec:PXD000561:Adult_Frontalcortex_bRP_Elite_85_f09:scan:17555:VLHPLEGAVVIIFK/2
Example 1: Peptide Spectrum Match (PSM) of an unmodified doubly-charged peptide VLHPLEGAVVIIFK from the Kim
et al. ${ }^{16}$ draft human proteome dataset. Most of the intense unannotated peaks are internal fragmentation ions of this
peptide.

Case 2: Flexible notation for reporting identification of post-translational modifications (one spectrum, interpretation with Unimod names, Unimod identifiers, PSI-MOD names and PSI-MOD identifiers for mass modifications)

\begin{tabular}{|c|}
\hline \\
\hline $\begin{array}{l}\text { mzspec:PXD000966:CPTAC_CompRef_00_iTRAQ_05_2Feb12_Cougar_11-10- } \\
\text { 09.mzML:scan:12298:[UNIMOD:214]-LHFFM[UNIMOD:35]PGFAPLTSR/2 } \\
\text { Example 2b: Same CPTAC PSM with modifications specified using Unimod accession numbers. This notation is } \\
\text { equally precise but not as easily readable as modification names. }\end{array}$ \\
\hline $\begin{array}{l}\text { mzspec:PXD000966:CPTAC_CompRef_00_iTRAQ_05_2Feb12_Cougar_11-10- } \\
\text { 09.mzML:scan:12298:[MOD:01499]-LHFFM[L-methionine sulfoxide]PGFAPLTSR/2 }\end{array}$ \\
\hline Example 2c: Same CPTAC PSM with modifications specified using PSI-MOD names an \\
\hline $\begin{array}{l}\text { mzspec:PXD000966:CPTAC_CompR } \\
\text { 09.mzML:Scan:12298:[+144.1021]-LH } \\
\text { Example 2d: Same CPTAC PSM with } n \\
\text { discouraged when the type of modificat } \\
\text { open modification searches returning al }\end{array}$ \\
\hline
\end{tabular}

Case 3: Supporting evidence of translated gene products (i.e., protein existence) as detected in public datasets, including matches to spectra of synthetic peptides as required by the HUPO Human Proteome Project (HPP) guidelines for detection of novel proteins

mzspec:PXD022531:j12541_C5orf38:scan:12368:VAATLEILTLK/2
Example 3a: Identification derived from a prey protein (Q5VTAO) in the Huttlin et al. ${ }^{18}$ BioPlex dataset, pulled down as
a binding partner to bait protein C5orf38. With only this single identification, this protein remains an HPP missing
protein since a single identification does not meet HPP guidelines.
mzspec:PXD022531:b11156_PRAMEF17:scan:22140:VAATLEILTLK/2
Example 3b: PSM of the same peptide as above, but derived from a recombinant protein used as a bait in the Huttlin et
al. BioPlex dataset. This PSM provides a much higher signal-to-noise ratio synthetic peptide reference spectrum as
required by HPP guidelines.

Case 4: Data reanalysis refuting previous claims of novel HLA (Human Leukocyte Antigen) peptides mzspec:PXD000394:20130504_EXQ3_MiBa_SA_Fib-2:scan:4234:SGVSRKPAPG/2
Example 4a: Identification originally used to reported a novel HLA peptide (Mylonas et al. ${ }^{4}$ Figure 2A).

mzspec:PXD010793:20170817_QEh1_LC1_HuPa_SplicingPep_10pmol_G2_R01:scan:8296:SGVSRKPAPG/2

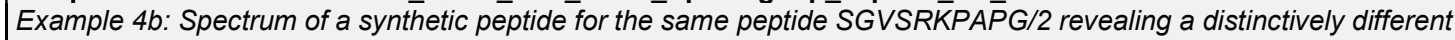
fragmentation pattern from example $4 a$.

mzspec:PXD000394:20130504_EXQ3_MiBa_SA_Fib-2:scan:4234:ATASPPRQK/2

Example 4c: The same spectrum as for example 4a, but correctly identified to commonly-occurring peptide from UniProtKB protein Q9UQ35 (Mylonas et al. Figure 2B).

mzspec:PXD010793:20170817_QEh1_LC1_HuPa_SplicingPep_10pmol_G2_R01:scan:7452:ATASPPRQK/2 Example 4d: Spectrum of a synthetic peptide for ATASPPRQK/2 (same as $4 c$ ) with a fragmentation pattern matching example 4c, thus confirming the identification to protein Q9UQ35.

Case 5: Reporting spectra of unidentified peptides with the potential to lead to interesting new discoveries mzspec:PXD010154:01284_E04_P013188_B00_N29_R1.mzML:scan:31291 Example 5a: Unidentified peptide detected by clustering as highly abundant only in Small intestine and Duodenum out of 29 human tissues in PXD010154. mzspec:PXD010154:01284_E04_P013188_B00_N29_R1.mzML:scan:31291:DQNGTWEM[Oxidation]ESNENFEG YM[Oxidation]K/2

Example 5b: Manual annotation of the spectrum from example 5a reveals it to be a multiply-modified version of a peptide previously detected only as unmodified. 